\title{
Droplet Splashing by a Slingshot Mechanism
}

\author{
S. T. Thoroddsen, ${ }^{1}$ M.-J. Thoraval, ${ }^{1}$ K. Takehara, ${ }^{2}$ and T. G. Etoh $^{2}$ \\ ${ }^{1}$ Division of Physical Sciences and Engineering and Clean Combusion Research Center, King Abdullah University of Science \\ and Technology, Thuwal 23955-6900, Saudi Arabia \\ ${ }^{2}$ Civil and Environmental Engineering, Kinki University, Higashi-Osaka 577-8502, Japan
}

(Received 1 August 2010; published 18 January 2011)

\begin{abstract}
When a drop impacts onto a liquid pool, it ejects a thin horizontal sheet of liquid, which emerges from the neck region connecting the two liquid masses. The leading section of this ejecta bends down to meet the pool liquid. When the sheet touches the pool, at an "elbow," it ruptures and sends off microdroplets by a slingshot mechanism, driven by surface tension. High-speed imaging of the splashing droplets suggests the liquid sheet is of submicron thickness, as thin as $300 \mathrm{~nm}$. Experiments in partial vacuum show that air resistance plays the primary role in bending the sheet. We identify a parameter regime where this slingshot occurs and also present a simple model for the sheet evolution, capable of reproducing the overall shape.
\end{abstract}

DOI: 10.1103/PhysRevLett.106.034501

PACS numbers: 47.55.D-

The splashing of a drop impacting onto a liquid layer is of great importance in a number of industrial applications, like combustion, spray painting, cleaning, and cooling, as well as natural processes such as air entrainment, aerosol formation through atomization, and erosion by rain [1]. Recent improvements in high-speed video imaging have started to allow characterization of the finer details of these processes [2]. This has revealed numerous intricate new fluid phenomena, not previously observed or understood. One of these is the emergence of a fine jet of fluid following the initial contact of the impacting drop with the pool liquid [3-6]. Despite their small size, these fine structures can have dramatic effects on the overall flow and the formation of spray through the breakup of the liquid edge.

Figure 1 shows details of the ejecta sheet and the slingshot mechanism, which splashes secondary microdroplets. The sheet emerges from the neck region between the drop and the pool, following the first contact. Then the sheet bends characteristically downwards and touches the pool (panel 4) at an elbow where the sheet ruptures (5). The thicker leading tip of the sheet provides a counterbalance to slingshot the ruptured end through pulling by the surface tension. This mechanism shoots microdroplets horizontally at high speed (7-10).

We use gravity-driven water-glycerin drops, from $0 \%$ to $90 \%$ glycerin, giving a viscosity range up to $\mu=109 \mathrm{cP}$. The drops are released from a range of nozzle diameters, generating drops with diameters $D$ from 3.2 to $6.0 \mathrm{~mm}$. The liquid pool is always deeper than $2 D$. The impact velocity $U$ is varied by changing the release heights up to $4.3 \mathrm{~m}$. The Reynolds, Weber, and Ohnesorge numbers of the impact are defined, respectively, as

$$
\operatorname{Re}=\frac{\rho D U}{\mu} ; \quad \mathrm{We}=\frac{\rho D U^{2}}{\sigma} ; \quad \mathrm{Oh}=\frac{\mu}{\sqrt{\rho \sigma D}},
$$

where $\mu$ and $\rho$ are the dynamic viscosity and density of the liquid, respectively, and $\sigma$ is the surface tension. Gravity plays no role on these very short time scales ( $<2 \mathrm{~ms}$ ). The $\mathrm{Re}$ and We are often rearranged to form the splashing parameter $[7,8]$, encompassing inertia, surface tension, and viscous effects:

$$
K=\mathrm{We} \sqrt{\operatorname{Re}}=\sqrt{\rho^{3} D^{3} U^{5} /\left(\sigma^{2} \mu\right)} .
$$

This parameter arises naturally, from the following mechanistic considerations, by forming a local $\mathrm{We}_{\ell}$ based on the relevant local length scale being the ejecta sheet thickness $\delta \sim \sqrt{\nu t} \sim \sqrt{\nu D / U}$ and using the ejecta velocity, which scales as $u_{j} \sim U \sqrt{\operatorname{Re}}$ based on Refs. [4,5]. By substitution, this produces $\mathrm{We}_{\ell}=\rho \delta u_{j}^{2} / \sigma=K$.

To capture the details of the rapid ejecta motions, we pursue a two-pronged approach, using both an ultrahighspeed video camera for sufficient temporal resolution and a dual-frame particle image velocimetry camera in combination with $\mathrm{Nd}$ :YAG pulsed laser sheet, for higher spatial

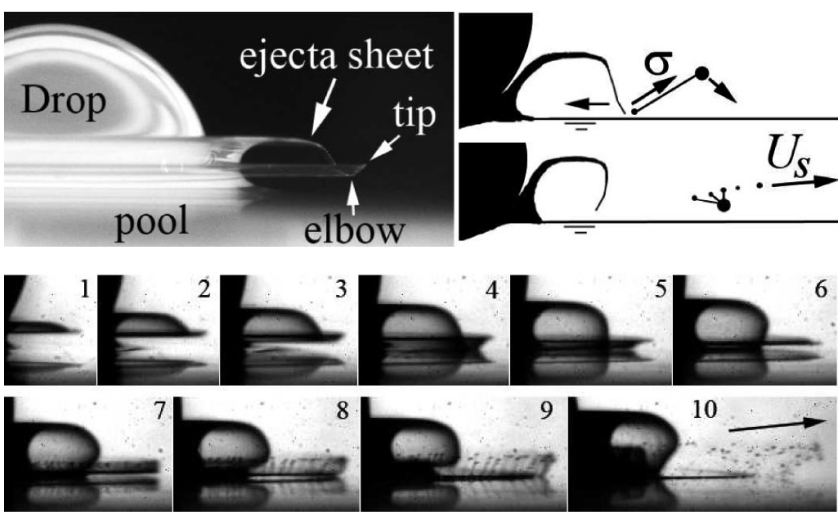

FIG. 1. (Top) Typical ejecta sheet and sketch showing the slingshot mechanism. (Bottom sequence) Ejecta sheet emerges, bends downwards, ruptures as it touches the pool, and then slingshots droplets horizontally. Frames are 50, 70, 80, 90, $100,110,120,130,140$, and $170 \mu$ s after impact. See Ref. [19]. 


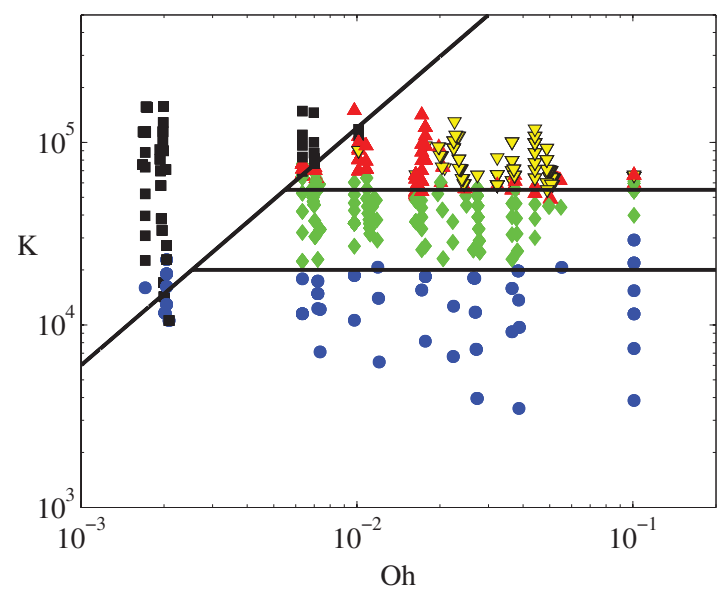

FIG. 2 (color). Characterization of the ejecta sheet dynamics. (ם) Irregular broken sheets and spray. (Blue $\bigcirc$ ) Ejecta sheets do not bend towards the pool. (Green $\diamond$ ) Sheets bend downwards but do not reach the pool. For both triangles, the sheet bends downward and reaches the pool. (Red $\triangle$ ) Sheet breaks before impacting the pool and no slingshot. (Yellow $\nabla$ ) Slingshot of fine droplets.

resolution [4]. The video camera [9] is capable of 1000000 frames/s, while each clip has 102 frames of $260 \times 312$ pixels. The time counter in the online video is in microseconds.

In Fig. 2, we show the region of parameter space where the ejecta sheets are formed and categorize their evolution. When the ejecta sheet does not break in fine droplets at ejection (at low $\mathrm{Oh}$ ), its evolution is clearly determined by the $K$ parameter. For $K<2 \times 10^{4}$ the sheet does not bend toward the pool. For $K$ between $2 \times 10^{4}$ and $5.5 \times 10^{4}$ it bends but does not reach the pool surface, whereas for $K>5.5 \times 10^{4}$ it touches the pool surface. In this case, for Oh $<2 \times 10^{-2}$ the tip of the sheet breaks before reaching the pool (red $\triangle$ ), while for larger Oh (yellow $\nabla$ ) the slingshot mechanism is observed; see Fig. 1. Deegan, Brunet, and Eggers [10] have similarly shown that $K$ scaling works for the crown breakup.

In the slingshot regime, the ejecta bends down sooner for higher values of $K$ and hits the pool closer to the impact center. When the sheet elbow touches the pool surface, we measure the height of the trapped toroidal tent of air, $H$ in the inset in Fig. 3 and in Fig. 6. This height scales very well with $K$ over a range of 3 different fluid viscosities and 2 drop diameters.

Figure 4 shows the characteristic velocity of the droplets $U_{s}$ which are slingshot horizontally. Their speed increases linearly with larger $K$ when $\mathrm{Oh}$ is kept constant $(\mathrm{Oh}=$ 0.049 , moving vertically in Fig. 2). The well-known Taylor-Culick law gives the translational velocity of a free liquid edge as $U_{\mathrm{TC}}=\sqrt{2 \sigma /(\rho \delta)}$, where $\delta$ is the thickness of the liquid sheet. However, the Taylor-Culick law refers to the velocity of the edge relative to the internal velocity in the sheet. While we expect the sheet to start

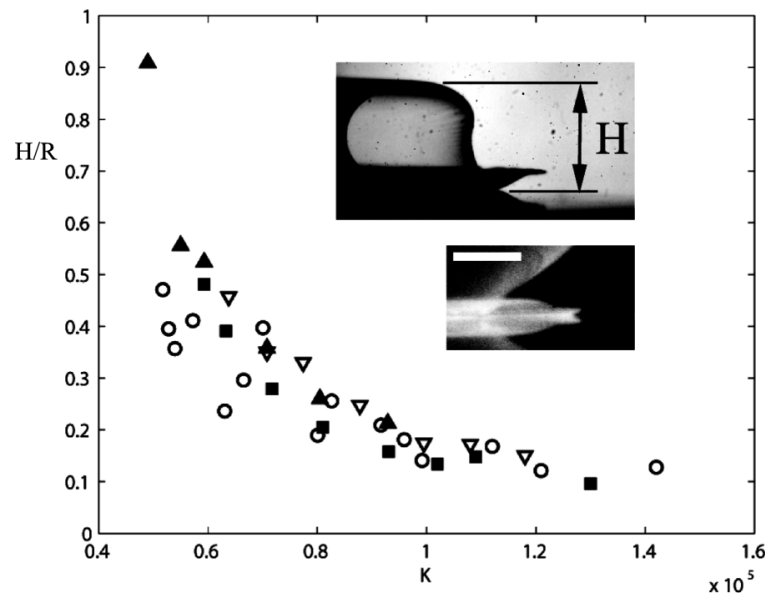

FIG. 3. The height of the ejecta sheet $H$ when the elbow touches the pool, normalized by the drop radius $R$, vs $K$. Data for $\mathrm{Oh}=0.017(\mu=10 \mathrm{cP}, D=4.4 \mathrm{~mm})(\bigcirc), 0.023(\mu=$ $14 \mathrm{cP}, D=4.4 \mathrm{~mm})(\square), 0.044(\mu=30 \mathrm{cP}, D=5.7 \mathrm{~mm})$ $(\nabla)$, and $0.049(\mu=30 \mathrm{cP}, D=4.4 \mathrm{~mm})(\mathbf{\Delta})$. Lower inset: The ejecta sheet for large value of $K$, where it touches the pool $\sim 50 \mu$ s after impact, when the drop has penetrated only $7 \%$ of $R$ into the pool. The bar is $0.5 \mathrm{~mm}$.

from rest as it ruptures, the slingshot further accelerates the edge as it approaches the tip, which is moving forward at $U_{\text {tip }}$. Therefore, we use $U_{\text {tip }}$ as an estimate of the sheet velocity and $U_{s}$ for the absolute retraction velocity of the film. We can thereby estimate $U_{\mathrm{TC}}=U_{s}-U_{\text {tip }}$ and thus the sheet thickness at the location where it ruptures, i.e., $\delta=2 \sigma / \rho\left(U_{s}-U_{\text {tip }}\right)^{2}$ (Fig. 4). This suggests that the sheet still remains intact while the film is as thin as $300 \mathrm{~nm}$ and ruptures only when it touches the pool. Figure 5 shows higher-resolution dual-frame images of the breakup of the sheet, where the film breaks up into tendrils, with thicker heads, which subsequently break up

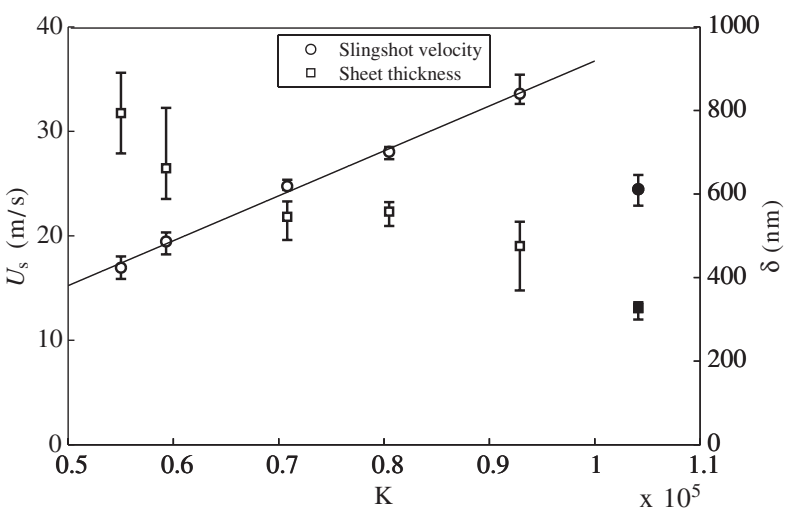

FIG. 4. Slingshot velocity $U_{s}$ of droplets and the corresponding film thickness $\delta$ based on the relative Taylor-Culick law. For $\mu=75 \mathrm{cP}$ and $\mathrm{Oh}=0.049$ (open symbols). Filled symbols show results for the largest impact velocity $U=7.8 \mathrm{~m} / \mathrm{s}$ and $\mu=109 \mathrm{cP}(\mathrm{Oh}=0.18)$. 


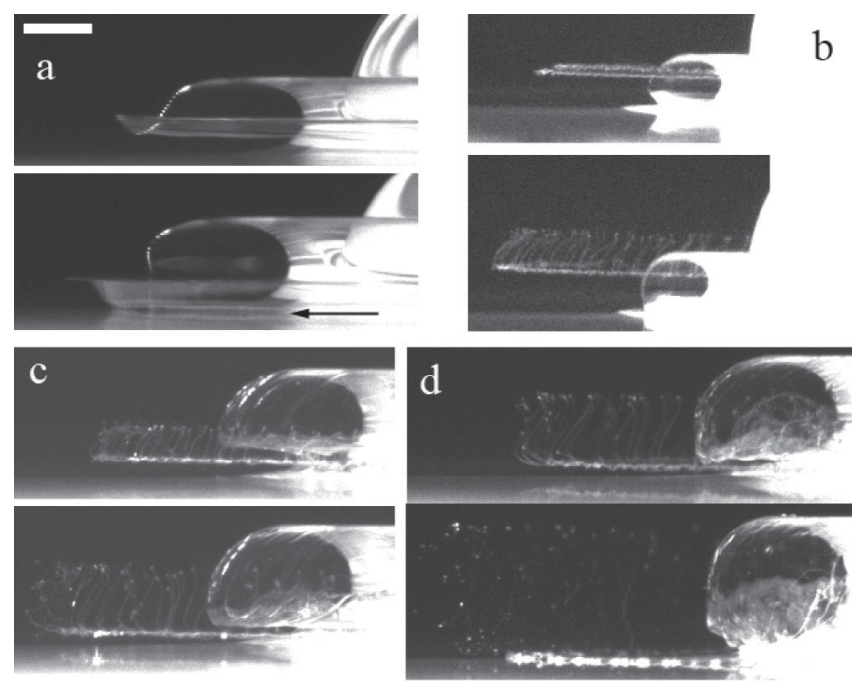

FIG. 5. Slingshotting sheets break up into tendrils and then droplets, $\operatorname{Re} \simeq 3500$. Times between image pairs ( $d t$ ) are (a) 50 , (b) 35, (c) 50, and (d) $100 \mu \mathrm{s}$. The arrow in lower panel of (a) points out the rupture of the film as it touches the pool. The bar is $1 \mathrm{~mm}$.

into a spray of microdroplets. Estimates of $\delta$ based on the tendril diameters also give submicron film thicknesses.

The bending of the sheet towards the pool is produced both by the kinematics of ejection, as is shown in the below model, but more importantly by air resistance. This was demonstrated by experiments in partial vacuum. Figure 6 compares two impacts, where only the air pressure $P_{g}$ is changed, by a factor of 5 , showing that at reduced air pressure the sheet does not bend downwards but rather rises away from the pool. Another indication of the effect of the air is that, as the elbow approaches the pool surface, it is strongly accelerated downwards, due to the enclosure of the rising torus of air, which in combination with incompressibility demands that air be pulled under the elbow. This generates Bernoulli suction pressure as the film closes to entrap the air torus [2,10], with a similar mechanism as the bubble separation described in Gordillo et al. [11] and Gekle et al. [12]. Figure 7 tracks the motion of the elbow next to the tip of the ejecta, over a range of air pressures, highlighting the central role of the air resistance.

The initial ejecta shown herein are characterized by highly curved shapes. Similarly curved forms can be produced by pure kinematics. This is perhaps most clearly demonstrated by a simple geometric model of a sheet ejected by a solid sphere of radius $R_{\mathrm{sp}}$ impacting onto a flat free surface with velocity $V_{\text {sp }}$; see the inset in Fig. 8(a). In this model the ejection velocity $V_{e}$ is assumed to be directed tangentially to the sphere at its intersection with the original liquid surface, with a speed proportional to the normal velocity of the sphere at this contact line, i.e., $V_{e}=C V_{\mathrm{sp}} \cos (\theta)$, where $C$ is the proportionality constant. Experimental [4] and theoretical results [5] show that

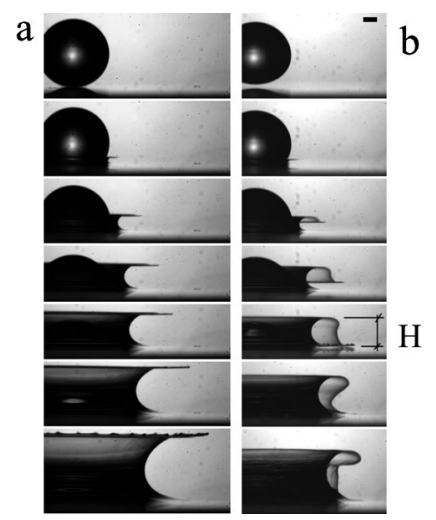

FIG. 6. The effect of air pressure on the ejecta shapes. Impact of a $73 \%$ glycerin-water drop $(D=5 \mathrm{~mm})$ onto a liquid layer, for different air pressures, for $\mathrm{Re} \simeq 820$ and $\mathrm{We}=2700$. (a) $P_{g}=0.21$ bar and (b) $P_{g}=1.0$ bar. Times shown are $\simeq 0$, $150,300,450,600,850$, and $1250 \mu \mathrm{s}$. The scale bar is $1 \mathrm{~mm}$.

$C=C^{\prime} \mathrm{Re}^{1 / 2}$, with $C^{\prime} \simeq 0.14$. If the initial contact occurs at time $t=0$ and the surface remains flat, then the location of the contact point moves outwards along the flat surface as $x_{c}(t)=\sqrt{V_{\mathrm{sp}} t\left(2 R_{\mathrm{sp}}-V_{\mathrm{sp}} t\right)}$. The radial velocity of the contact point

$$
V_{c}(t)=\frac{d x_{c}}{d t}=\frac{V_{\mathrm{sp}}\left(R_{\mathrm{sp}}-V_{\mathrm{sp}} t\right)}{\sqrt{V_{\mathrm{sp}} t\left(2 R_{\mathrm{sp}}-V_{\mathrm{sp}} t\right)}} \propto \frac{1}{\sqrt{t}}
$$

is initially infinite, dictated by the sphere geometry. The jetting velocity, in the model, is however finite: $C V_{\mathrm{Sp}}$. A more realistic model suggests that the sheet emerges only when this velocity exceeds the outwards motion of the contact point. This occurs when $V_{e}=V_{c}$, i.e., at $t_{e} \simeq$ $R_{\text {sp }} / 2 C^{2} V_{\text {sp }}$. This point is marked by a bead on the curve, giving the initial angle of ejection. By pure kinematics we let each fluid element move in a straight line after being

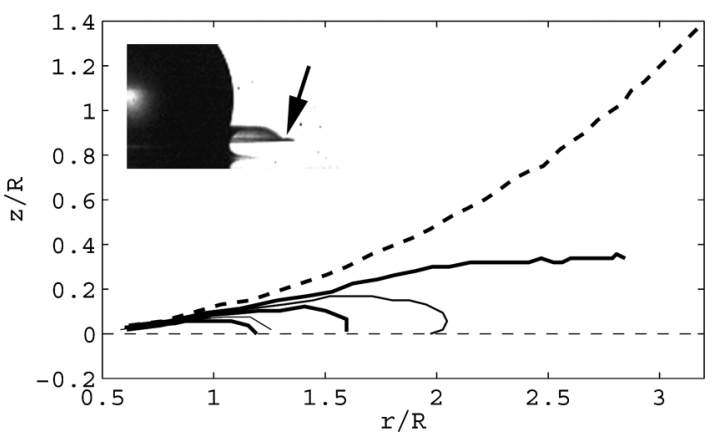

FIG. 7. The effect of air pressure on the trajectory of the edge of the ejecta sheet. We follow the elbow just behind the tip, as this point touches the pool first. The curves correspond to air pressures of (from bottom to top curve) $P_{g}=1.0,0.74,0.47$, $0.34,0.28,0.14$ bar (broken line). Data are for $58 \%$ glycerinwater mixture at $\mathrm{Re}=2450$ and $\mathrm{We}=2700$. The distances are normalized by the drop radius. 
a

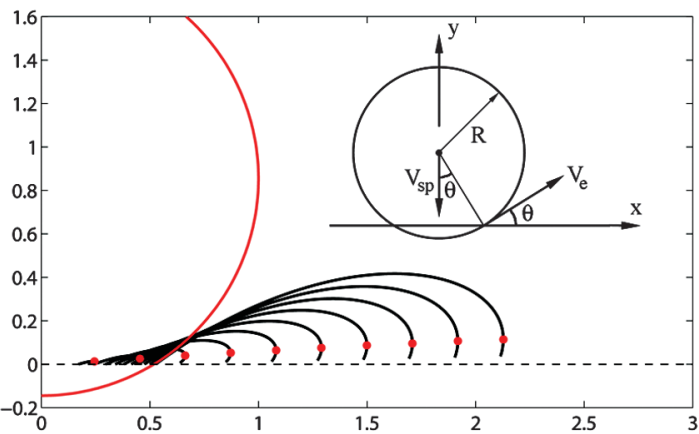

b

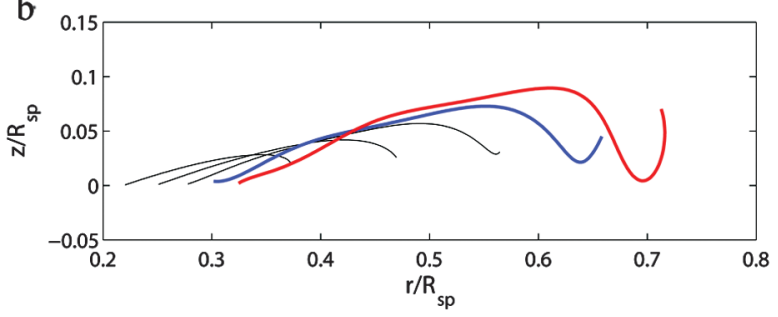

FIG. 8 (color). (a) Kinematic model of the sheet evolution for $C=14$, plotted for $\Delta \tau=0.015$. (b) Evolution of the curved liquid sheet, subjected to air resistance, but ignoring viscous stress and surface tension, for $R_{\mathrm{sp}}=2.5 \mathrm{~mm}, V_{\mathrm{sp}}=4 \mathrm{~m} / \mathrm{s}$, and $\delta=30 \mu \mathrm{m}$. The drag coefficient is fixed at $C_{d}=2$. The tip is kept slightly thicker to mimic the observed bead produced by surface tension. Shapes shown until $200 \mu$ s.

ejected. The shape of the ejected sheet can now be observed at time $\tau$ akin to the way one describes a streak line in unsteady flow, with $t$ as the parameter to identify each fluid element. The location of fluid element ejected at $t$ and observed at time $\tau$ is

$$
\begin{gathered}
x(t, \tau)=x_{c}(t)+V_{e} \cos (\theta)(\tau-t), \\
y(t, \tau)=V_{e} \sin (\theta)(\tau-t),
\end{gathered}
$$

where $\theta$ is a function of $t$ and $t<\tau$. Next we substitute for $V_{e}$, and without loss of generality we normalize the space coordinates by $R_{\mathrm{sp}}$, the velocity by $V_{\mathrm{sp}}$, and the time by $R_{\mathrm{sp}} / V_{\mathrm{sp}}$. These equations thereby become

$$
\begin{gathered}
x(t, \tau)=\sqrt{t(2-t)}+C(1-t)^{2}(\tau-t), \\
y(t, \tau)=C(1-t) \sqrt{t(2-t)}(\tau-t) .
\end{gathered}
$$

This produces a variety of space curves, which help explain why the sheet appears to bend towards the pool. Figure 8 shows kinematic shapes for a few values of $\tau$ and a fixed $C$. Related results have been obtained by Peregrine [13] for circular jet impacting a shallow liquid layer. However, the experiments in partial vacuum show that the air resistance is instrumental in promoting catastrophic bending, when considering the air resistance for motions perpendicular to the sheet. This is shown in Fig. 8(b), where we add air resistance to the component of the motion which is normal to the sheet $U_{n}$, based on high Re form drag $\propto U_{n}^{2}$, giving normal deceleration $\propto \rho_{\text {air }} U_{n}^{2} /(\rho \delta)$. Using the same initial ejecta conditions, when we integrate the motions including this drag, we see the axisymmetric sheet deform into shapes more similar to the experiments.

In this Letter, we investigate a new mechanism of microdroplet splashing through rupturing of ejecta sheets, which differs from earlier mechanisms [1,14-17] and produces much smaller droplets than the typical crown breakup. The influence of the air pressure arises here through changes in the gas density and not by the compressibility of the air, as proposed by Xu, Zhang, and Nagel [18] for droplet splashing on a solid surface. This is clear as the value of the Mach number for the ejecta sheet is $\mathrm{Ma} \sim 0.1$ and the fact that compressibility effects scale as $\mathrm{Ma}^{2}$. The gas pressure does not affect the emergence of the ejecta sheet which is determined by the early dynamic pressure inside the liquid, but greatly effects the bending of the sheet. It remains to be determined how the gas density $\rho_{g} \propto P_{g}^{-1}$ can be incorporated into the nondimensional description of the phenomenon. Keep in mind that the gas dynamic viscosity is insensitive to the air pressure. Numerous other intriguing but robust ejecta shapes have been observed (e.g., Fig. 8 in [2]) which require further study to shed new light on the dynamics of rapidly stretched submicron sheets of liquid.

[1] A. L. Yarin, Annu. Rev. Fluid Mech. 38, 159 (2006).

[2] S. T. Thoroddsen, T. G. Etoh, and K. Takehara, Annu. Rev. Fluid Mech. 40, 257 (2008).

[3] D. A. Weiss and A.L. Yarin, J. Fluid Mech. 385, 229 (1999).

[4] S. T. Thoroddsen, J. Fluid Mech. 451, 373 (2002).

[5] C. Josserand and S. Zaleski, Phys. Fluids 15, 1650 (2003).

[6] S. D. Howison et al., J. Fluid Mech. 542, 1 (2005).

[7] C. D. Stow and M. G. Hadfield, Proc. R. Soc. A 373, 419 (1981).

[8] C. Mundo, M. Sommerfeld, and C. Tropea, Int. J. Multiphase Flow 21, 151 (1995).

[9] T. G. Etoh et al., IEEE Trans. Electron Devices 50, 144 (2003).

[10] R. D. Deegan, P. Brunet, and J. Eggers, Nonlinearity 21, C1 (2008).

[11] J. M. Gordillo et al., Phys. Rev. Lett. 95, 194501 (2005).

[12] S. Gekle et al., Phys. Rev. Lett. 104, 024501 (2010).

[13] D. H. Peregrine, J. Fluid Mech. 106, 59 (1981).

[14] R. Rioboo, C. Tropea, and M. Marengo, At. Sprays 11, 155 (2001).

[15] I. V. Roisman, K. Horvat, and C. Tropea, Phys. Fluids 18, 102104 (2006).

[16] E. Villermaux, Annu. Rev. Fluid Mech. 39, 419 (2007).

[17] H. Lhuissier and E. Villermaux, Phys. Rev. Lett. 103, 054501 (2009).

[18] L. Xu, W. W. Zhang, and S. R. Nagel, Phys. Rev. Lett. 94, 184505 (2005).

[19] See supplementary material at http://link.aps.org/ supplemental/10.1103/PhysRevLett.106.034501 for video clips. 